\title{
Osteopathie bei epileptischen Anfällen
}

\author{
Edgar Hinkelthein
}

\author{
Bei einem Jugendlichen mit einer symptomatisch-fokalen Epilepsie mit generalisierten \\ tonisch-klonischen Anfällen und dyskognitiven Anfällen konnte die Anfallshäufigkeit medi- \\ kamentös nicht reduziert werden, sodass nach 2,5 Jahren eine Hirn-Teilresektion geplant \\ war. Mittels osteopathischer Therapie konnte innerhalb von 5 Monaten eine bleibende \\ Anfallsfreiheit erreicht werden, die seit mehr als einem Jahr anhält.
}

\section{Kasuistik}

\section{Anamnese}

Bei dem 15-jährigen Patienten kam es im April 2016 zum 1. epileptischen Anfall. Wegen persistierender Anfälle erhielt er verschiedene Medikamente. Dennoch traten mehrfach pro Woche, besonders in Stresssituationen, wechselnd stark ausgeprägte, überwiegend dyskognitive Anfälle mit Bewusstseinsverlust, starrem Blick und häufig anschließendem Nachschlaf auf. Die Anfälle ereigneten sich zu wechselnden Tageszeiten, auch unter der begonnenen ketogenen Diät bestanden weiterhin Anfälle. Im Wach-EEG mit Hyperventilation zeigte sich ein kombinierter Herdnachweis rechts temporal in Form einer intermittierenden Verlangsamung mit Sharp Waves und Sharp Slow Waves. Im MRT ließ sich ein Kavernom im rechten Parietallappen nachweisen.

Die Diagnose lautete: symptomatisch-fokale Epilepsie mit generalisierten tonisch-klonischen Anfällen und dyskognitive Anfälle mit und ohne Adversivbewegung des Kopfes bei z. B. Einblutung eines Kavernoms rechts parietal.

Wegen der Therapieresistenz war eine Hirnoperation mit Teilresektion geplant.

\section{Befund und Behandlung}

Bei der Vorstellung in meiner Praxis 2,5 Jahre nach dem 1. Anfall lagen neben den beschriebenen epileptischen Anfällen auch rezidivierende Rückenschmerzen mit Bewegungseinschränkungen vor. Bei der Untersuchung fanden sich neben Dysfunktionen der Beckengelenke (Sakroiliakalgelenk und Iliosakralgelenk) mit adaptiven Funktionsstörungen v. a. im BWS-Bereich auch Blockaden der Handgelenke sowie ein Atlas-Shift nach links. Viszeral war der Befund unauffällig, kraniosakral waren die horizontale und vertikale Dura gespannt und somit auch die Beweglichkeit beider Ossa temporalia eingeschränkt. Außerdem zeigte sich eine kraniale venöse Kongestion.

Je nach erhobenem Befund erfolgte die osteopathische Behandlung. Während der ersten 4-5 Behandlungen traten die Anfälle statt 2-3-mal pro Woche nur noch alle 23 Wochen auf. Nach jedem Anfall lagen wiederum Blockaden der mittleren BWS und ein Atlas-Shift links mit gleichem kraniosakralen Ausgangsbefund vor. Da die Pathophysiologie eines epileptischen Anfalls - wenn auch mit Sturz - nicht den Erfordernissen für ein Atlas-Shift entsprach (seitlicher Anprall an Atlas oder Schädel), vereinbarten wir alle 2-3 Tage Kontrolluntersuchungen. Es zeigten sich wiederholt BWS-Blockaden, nur selten eine Atlas-Hypomobilität links. Der Patient nahm Apydan ${ }^{\circledR}$ extent und Lamotrigin ein.

5 Monate nach der Erstbehandlung lag eine Anfallsfreiheit vor, die nun seit 1,5 Jahren besteht. Mittlerweile kommt der Patient alle 4-6 Wochen zur Kontrolluntersuchung und ggf. werden Blockaden (meist 1-2 in der oberen BWS) gelöst. Kraniosakral sowie viszeral jeweils unauffälliger Befund.

Bei den neuropädiatrischen Kontrolluntersuchungen war jeweils das EEG mit Hyperventilation unauffällig (kein sicherer Herdnachweis, keine umschriebene Verlangsamung, keine epilepsietypischen Potenziale). Es wurde die Wiederaufnahme von Sport unter Beibehaltung der aktuellen Medikation empfohlen, da in der Vergangenheit Sport auch als Auslöser aufgetreten war. Abzuwarten bleibt das Kontroll-MRT, um die Entwicklung des diagnostizierten Kavernoms im rechten Parietallappen zu kontrollieren, nachdem im EEG-Befund der Herdbefund nicht mehr nachweisbar war. 


\section{Schulmedizinische Überlegungen}

Nach schulmedizinischem Wissen entstehen Epilepsien aufgrund abnormer Reaktionen von Nervenzellen. Diese Nervenzellen der grauen Substanz des Großhirns produzieren in rhythmischer Folge Aktionspotenziale (AP), die sowohl unsinnig (ohne Funktion) und viel zu stark ausgeprägt sind als auch endlos lange anhalten. Benachbarte "gesunde“ Nervenzellen werden von den Nervenzellen mit epileptischer Aktivität aufgrund deren erhöhter APIntensität „angesteckt“ und geben so die epileptische Aktivität in ungebremstem und übersteigertem Maße an immer weitere Nervenzellen weiter. Auf diese Art kann sich die epileptische Aktivität über das gesamte Großhirn ausbreiten; es tritt ein Grand-Mal-Anfall auf, bei dem sich das gesamte Gehirn in gleichgeschalteter, verstärkter, sinnloser Aktivität befindet. Hemmende Neurone können eine solche Ausbreitung epileptischer Aktivität beenden, indem sie die Aktivität der betroffenen Nervenzellen bremsen und (wie einen Computer) neu „hochfahren“, sodass die Nervenzellen wieder ihre normale Tätigkeit aufnehmen können. Auch die initial epileptischen Neuronen werden gebremst, allerdings starten sie wiederum mit einer epileptischen Aktivität, was durch bremsende Neurone nicht verhindert wird.

Man unterscheidet fokale und generalisierte Anfälle: Fokale beginnen in einer umschriebenen Region einer Großhirnhälfte, bei generalisierten sind keine Zeichen eines lokalen Beginns zu erkennen. Die dyskognitiven Anfälle beginnen fokal, breiten sich aber soweit aus, dass es zu Bewusstseinsbeeinträchtigungen kommt (keine Bewusstlosigkeit, aber inadäquate Wahrnehmung und
Handlung. Bei dyskognitiven Anfällen werden Temporallappenanfälle (mit Aura, starrer Motorik, Automatismen, inadäquate Reaktionen, vegetative Begleitsymptome) und Frontallappenanfälle (Bewusstseinsbeeinträchtigung mit [grob-]motorischen Automatismen) unterschieden.

Medikamentös werden verschiedene Antiepileptika (AE) eingesetzt, deren Wirkung darauf beruht, das Auftreten von Anfällen infolge der epileptischen Neurone zu unterdrücken. Die Wirksamkeit einzelner AE ist nicht vorauszusagen, sodass diese nacheinander solange auf ihre konkrete Wirkung am einzelnen Patienten ausprobiert werden müssen, bis man ein für diesen Patienten wirksames $A E$ gefunden hat. Oft müssen auch mehrere $A E$ kombiniert werden, um ein befriedigendes Ergebnis zu erhalten. Bei ca. 70\% der Epileptiker erreicht man eine Anfallsfreiheit, während ca. 30\% trotz Medikation therapieresistente epileptische Anfälle erleiden. Bei Temporallappenepilepsien liegt die Erfolgsquote unter $50 \%$, weswegen man neurochirurgisch versucht, eine Anfallsreduktion bzw. -freiheit zu erreichen.

Es gibt Zentren, die zur Behandlung von Epileptikern eine ketogene Diät empfehlen, weil die Anfallshäufigkeit durch Fasten vermindert wird. Eiweiße und Kohlenhydrate $(\mathrm{KH})$ werden weitgehend gemieden, während der Fettanteil der Nahrung erheblich gesteigert wird (Fett: $\mathrm{KH}=$ ca. 4:1). Studien zufolge werden ca. $30 \%$ der Patienten mit einer ketogenen Diät anfallsfrei, mehr als $50 \%$ der Patienten haben eine Minderung der Anfallshäufigkeit um mehr als die Hälfte. Allerdings reicht bereits eine einzelne kohlenhydratreiche „Diätsünde“ aus, um Anfälle wieder auftreten zu lassen. 


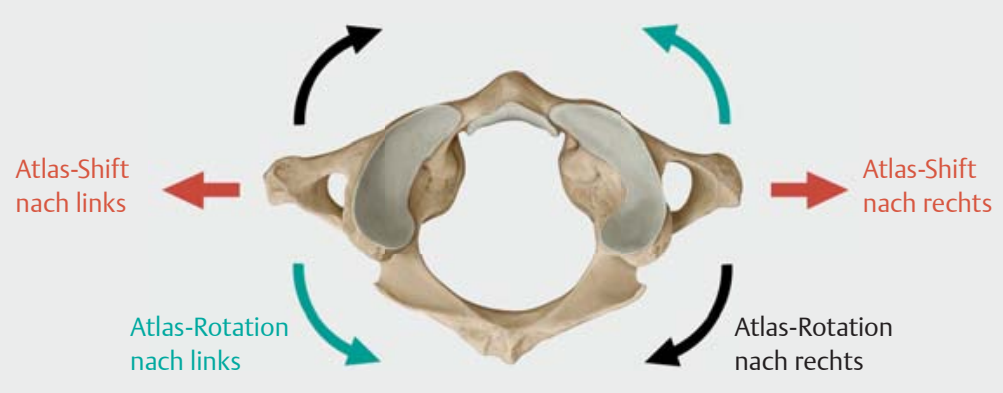

Abb. 1 Atlas-Shift. (Quelle: [2])

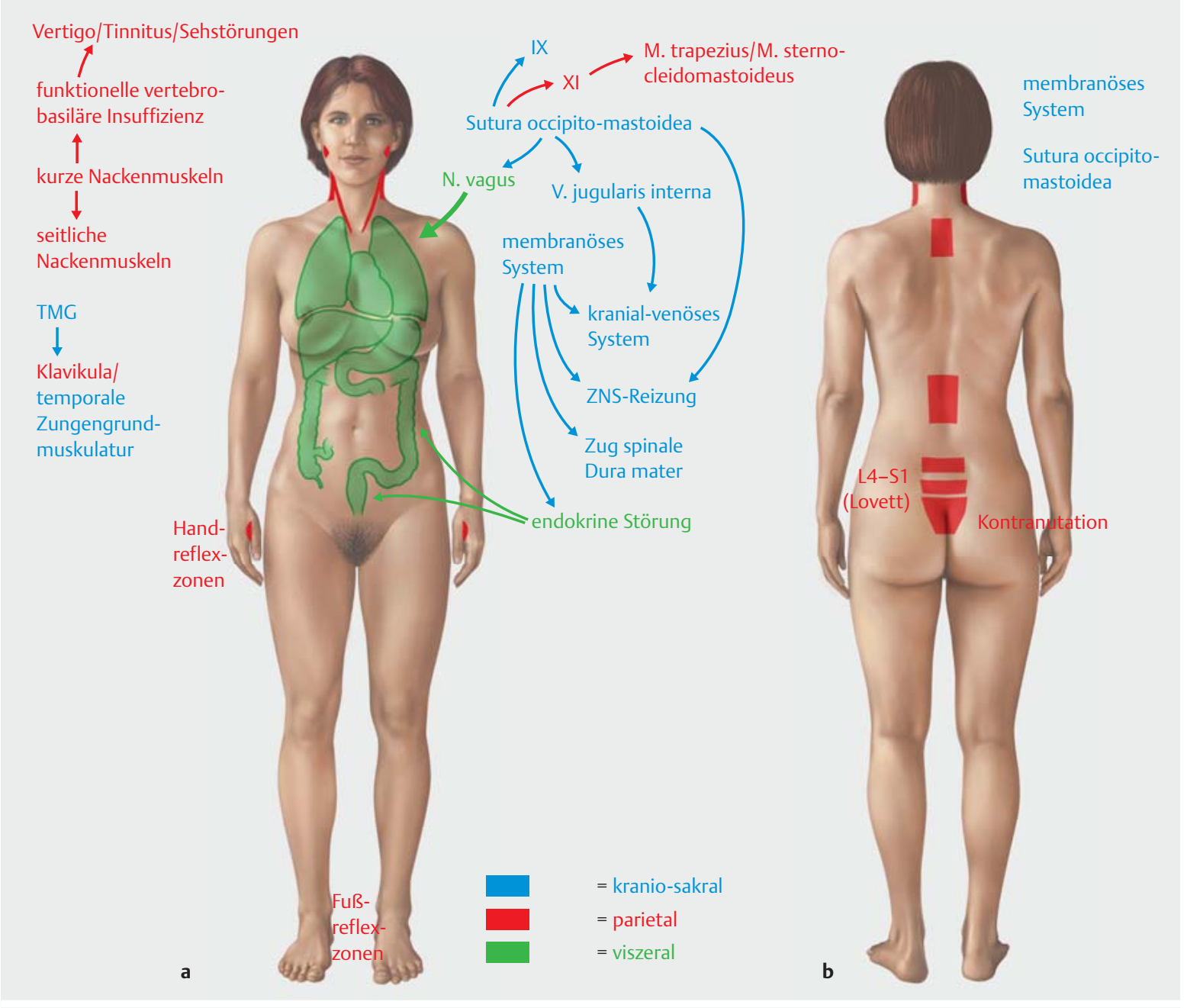

- Abb. 2 Folgen einer Dysfunktion des Okziput-Atlas-Axis-Komplexes. (Quelle: [2])

Kavernome sind umschriebene Gefäßfehlbildungen aus der Gruppe der Hämangiome, also dem kapillären Niederdrucksystem. Galten sie früher als extrem selten, werden sie mittels Kernspintomografie (MRT) immer häufiger entdeckt; man geht von 0,5\% Betroffenen innerhalb der Bevölkerung aus, in Deutschland also von ca. 400000
Menschen [1]. Nach Studien können 20-40\% der neurochirurgisch behandelten intrakranialen Blutungen auf Kavernome zurückgeführt werden und weniger auf klassische arteriovenöse Angiome. Studien zeigen eine häufigere Verbindung von epileptischen Anfällen mit zerebralen Kavernomen im Vergleich zu anderen vaskulären 


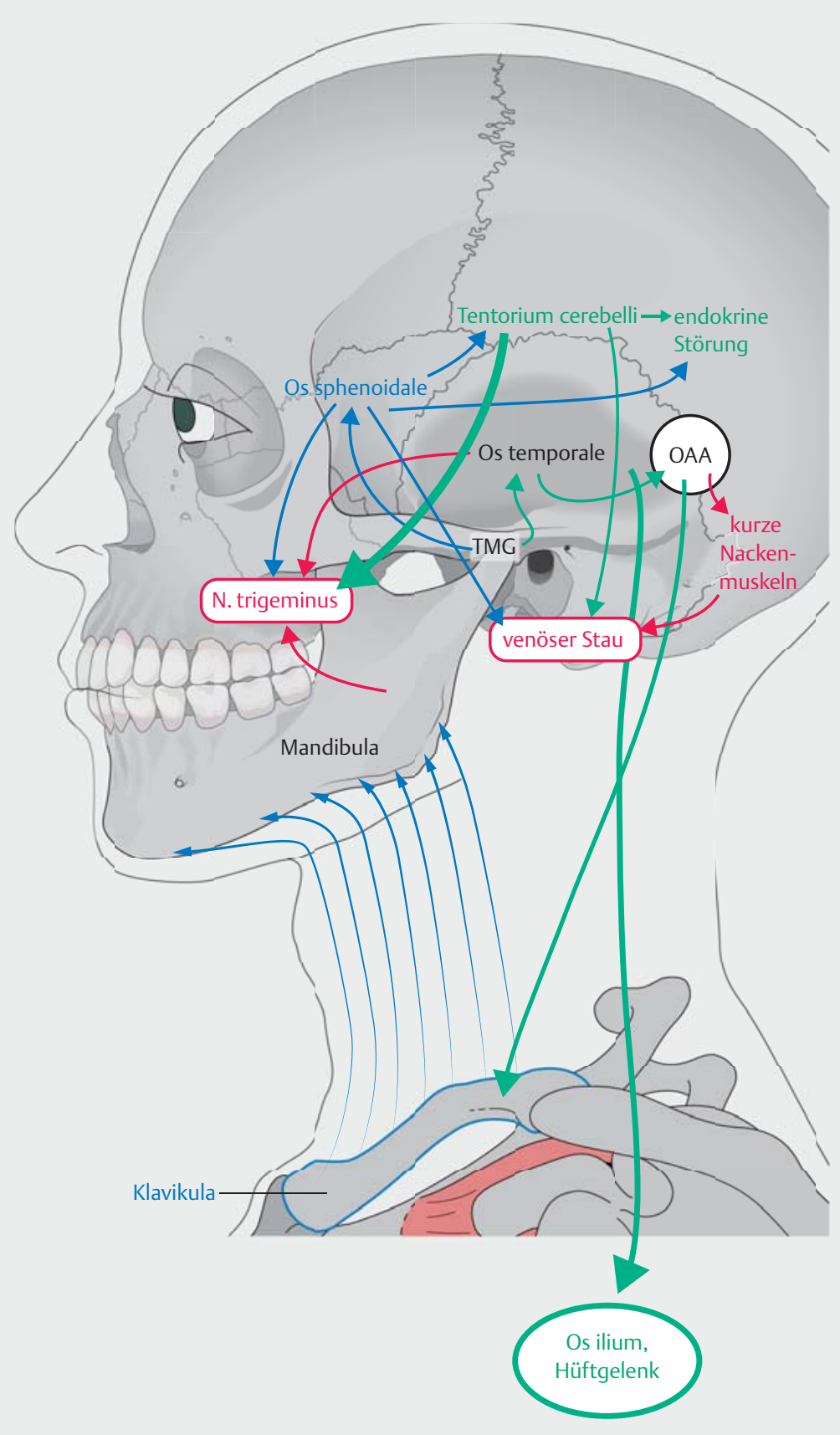

- Abb. 3 Folgen von TMG-Dysfunktionen. (Quelle: [2])

Abnormalitäten, was als epileptogenes Potenzial der Kavernome bezeichnet wird [1]. Die durch Mikroblutungen bedingten Ablagerungen wie hochepileptogene Eisensalze und die lokale gliomatöse Umgebungsreaktion werden als dafür verantwortlich diskutiert. Das prospektive Anfallsrisiko wird mit 4,8\% pro Patientenjahr mit einer durch Kavernome neu auftretenden Anfallsfrequenz von 2,4\% pro Patientenjahr beschrieben. Empfehlungen der International League Against Epilepsy (ILAE) für kavernombedingte Epilepsien lauten, bei einmaligem Anfall medika- mentös zu therapieren, bei den medikamentös nicht kontrollierbaren Formen immer präoperativ epileptologisch abzuklären.

\section{Osteopathische Überlegungen}

Aus osteopathischer Sichtweise liegen die im Folgenden beschriebenen 2 Dysfunktionskomplexe mit Bezug zur epileptischen Symptomatik vor. 


\section{Atlas-Shift}

Auswirkungen des Atlas-Shift ( $\bullet$ Abb. 1) links:

- parietal:

- hebt die physiologische Biomechanik der HWS auf

- nimmt dem Kopf die Kompensationsfähigkeit auf parietale Dysfunktionen des Körpers

- beeinflusst über die kurzen Nackenmuskeln

- die arterielle Versorgung der A. vertebrobasilaris für Innenohr, Gleichgewichtsorgan und Sehbahn

- das Foramen jugulare und damit eine venöse Kongestion der V. jugularis

- die seitlichen Halsmuskeln

- beeinflusst die Hand- und Fußreflexzonen (Daumen und Großzehe)

- beeinflusst die Übergänge der Wirbelsäule (kraniozervikal, zervikothorakal, thorakolumbal und lumbosakral) ( A Abb. 2)

- beeinflusst über die Lovett-Regel L5

- viszeral: beeinflusst

- die Sutura occipitomastoidea und damit die vegetative Innervation über N. vagus $(X)$ und N. glossopharyngeus (IX)

- über den linken N. vagus den AV-Knoten und über den rechten N. vagus den Sinus-Knoten und somit die Herzfrequenz

- kraniosakral: beeinflusst

- die Spannung der kranialen und spinalen Dura mater und das membranöse System mit Druck auf das Großhirn

- die Sutura occipitomastoidea

- das Temporomandibulargelenk (TMG) ( $\bullet$ Abb. $\mathbf{3}$ )

- die kraniosakralen Sakrum-SSB-Dysfunktionen

- den kranialen venösen Abfluss über die V. jugularis

\section{Spannung der horizontalen und vertikalen Dura}

Die kraniosakrale Spannung der horizontalen und vertikalen Dura mit Einschränkung der Beweglichkeit beider Ossa temporalia und der kranialen venösen Kongestion beeinflusst:

- Druck auf das Großhirn

- Sutura occipitomastoidea

- TMG

- kraniosakrale Sakrum-SSB-Dysfunktionen

- kranialen venösen Abfluss über die V. jugularis

Im Verlauf der Behandlung und bei den Kontrollen stellte sich heraus, dass die Dysfunktion des Atlas-Shift - wie bereits biomechanisch anzunehmen war - nicht die Folge, sondern die Ursache der epileptischen Anfälle des Patien- ten war. Die kraniosakralen Dysfunktionen, die auch epileptische Anfälle auslösen können, zeigten sich im Therapieverlauf als bleibend korrigiert, dennoch hatte jeder erneute Atlas-Shift einen epileptischen Anfall zur Folge. Somit dominierte in diesem Fall der Atlas-Shift auch die kraniosakralen Dysfunktionen. Darüber hinaus zeigten sich wiederholte Blockaden der BWS mit verstärkter Kyphose. Diese sind wahrscheinlich auf die Sportabstinenz und die vermehrte Nutzung von Tablet und Spielkonsole zurückzuführen, während der Patient auf dem Sofa saß und dabei gelegentlich einschlief, wobei eine schräge Nickbewegung des Kopfes nach vorne seitlich als auslösender Moment für einen Atlas-Shift fungierte.

Interessant war, dass unter der Therapie mit AE konstant ein EEG-Herdbefund vorlag, der sich nach osteopathischer Behandlung in einen Normbefund wandelte. Abzuwarten bleibt, ob der radiologische Befund eines Kavernoms mit Hämosiderinumrandung im Kontroll-MRT noch vorhanden sein wird.

Autorinnen/Autoren

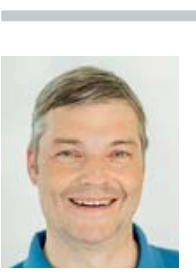

\section{Edgar Hinkelthein}

ist Facharzt für Orthopädie, Naturheilverfahren und Sportmedizin und Schulleiter der Akademie für Osteopathie und Naturheilverfahren (AON gGmbH).

Korrespondenzadresse

Dr. med. Edgar Hinkelthein B. Sc. (hons) Osteopath.

Med. (GB), D.O. M.R.O.

Satower Weg 29

24357 Fleckeby

Dr.Hinkelthein@t-online.de

Literatur

[1] Neubauer BA et al. Epilepsie im Kindes- und Jugendalter. Dt Ärzteblatt 2008; 105 (17): 319-328

[2] Hinkelthein E, Weitendorff A. Biomechanik in Osteopathischer und Manueller Medizin. Stuttgart: Thieme; 2019

Bibliografie

DOI https://doi.org/10.1055/a-1205-2240

DO - Deutsche Zeitschrift für Osteopathie 2020; 18: 18-22

(c) Georg Thieme Verlag KG Stuttgart · New York

ISSN 1610-5044 